\title{
The Effect of Time and Three Different Storage Environments on the Dimensional Stability of Acrylic Removable Orthodontic Appliances
}

\author{
Seyed Mohammad Mousavi ${ }^{1}$, Parinaz Saeidi Ghorani ${ }^{2}$, \\ Pedram Javidi ${ }^{3}$, Nazanin Berahman ${ }^{3}$ and Morteza Moattari ${ }^{4}$ \\ ${ }^{1}$ Assistant Professor, Department of Orthodontics, Faculty of Dentistry, \\ Ahvaz Jundishapur University of Medical Sciences, Ahvaz, Iran \\ ${ }^{2}$ Orthodontist. \\ ${ }^{3}$ Postgraduate Student of Orthodontics, Faculty of Dentistry, \\ Ahvaz Jundishapur University of Medical Sciences, Ahvaz, Iran. \\ 4 Postgraduate Student of Endodontics, Faculty of Dentistry, \\ Tehran Azad University of Medical Sciences, Tehran, Iran.
}

DOI: http://dx.doi.org/10.13005/bbra/1907

(Received: 10 August 2015; accepted: 16 October 2015)

\begin{abstract}
Acrylic resin is widely used in manufacturing removable orthodontic appliances. Nevertheless water sorption and dimensional changes are some of the drawbacks of this material that can affect the accuracy of the appliance. The aim of this study was to evaluate the effects of Corega ${ }^{\circledR}$ solution on dimensional changes of orthodontic appliances in comparison with two conditions including dry and water environment. Sixty six acrylic resin specimens were made in quadrangular metal mold. Then the length, width and diameter of each specimen were measured with digital caliper with measurement accuracy of $0.01 \mathrm{~mm}$. Specimens were randomly divided into three groups. The samples in these three groups were kept in dry environment, water and Corega ${ }^{\circledR}$ solution respectively. Different dimensions of the specimens were blindly measured after 18 hours, 48 hours and 1 week by two independent evaluators. Dry environment had the highest amount of dimensional change and was significantly different from water and Corega ${ }^{\circledR}$ solution over those three evaluated periods of time. No significant difference was seen between dimensional changes in water and Corega ${ }^{\circledR}$ solution. Also data analysis showed that acrylic resin's dimensional change in dry environment over different periods of time is not statistically significant. Orthodontic appliances should be kept in water or Corega ${ }^{\circledR}$ solution and Corega ${ }^{\circledR}$ solution is the superior choice due to its antibacterial effects.
\end{abstract}

Key words: Acrylic resin, Removable orthodontic appliance, Corega ${ }^{\circledR}$.

Acrylic resins were introduced to dentistry in 1937. To date no other material has been found that would imitate underlying soft tissue as good as acrylic resins. Low cost, ease of manipulation and acceptable physical properties make acrylic resins a good choice in fabricating

\footnotetext{
* To whom all correspondence should be addressed. Tel.: +9861-33205168; Fax: +9861-33205320; E-mail:pjavidi@gmail.com
}

removable orthodontic appliances, removable prosthetics, etc. ${ }^{1}$ On the other hand, function of the appliance depends on its adaptation with underlying tissue. Good adaptation increases the stability and retention of the appliance which results in better compliance.

Accuracy and adaptation of resin appliances depends on many factors such as method of fabrication, type of material, and its dimensional stability. ${ }^{2}$ Complex interactions between acrylic resin's surface area, volume and 
shape cause dimensional changes during construction and in service which is the biggest disadvantage of acrylic resins. ${ }^{3}$

Dixon et al. ${ }^{4}$ have reported that certain amount of dimensional change should be considered as one of the drawbacks of acrylic resins. Two types of dimensional change known as shrinkage and expansion can be seen in all acrylic resins. The reported linear shrinkage varies between 0.26-1.2\%. Acrylic appliances also tend to absorb water (called hygroscopic expansion). Little amount of water sorption in polished appliances can improve the dimensional stability. However, this hygroscopic expansion may compensate the shrinkage partially or completely or even exceed the initial size.These changes are more significant when a long term hiatus occurs in wearing the appliance. ${ }^{1}$

Resin appliances can become contaminated by fungi, Candida Albicans or bacterial plaque. To reduce such contaminations daily use of chemical disinfectants such as Glutaraldehyde, Sodium hypochlorite and Iodoform is recommended. ${ }^{5}$

Adverse effects of dental plaque on periodontal health and decay formation is comprehensively evaluated and documented. Removable orthodontic appliances used either as a retention appliance or as an active device, increase plaque accumulation on teeth surfaces, clasps, springs and acrylic base. ${ }^{6}$

Zabokova-Bilbilova et al. ${ }^{7}$ reported high occurrence of enamel demineralization white spots in 50 percent of patients undergoing orthodontic treatments for 2 years.

Removable orthodontic appliances can change the normal oral microbial flora into the pathologic microorganisms therefore meticulous hygienic care should be considered in orthodontic patients.

Long term water adsorption of polymethyl acrylates helps the microbial growth in surface porosities of acrylic appliances. ${ }^{6}$

Mechanical cleaning with a tooth brush is usually just enough to remove loosely attached food debris. Whereas microorganisms such as Candida Albicans can penetrate even polished acrylic bases into the depth of $1-2 \mu \mathrm{m} .{ }^{8}$

Lessa et al. ${ }^{6}$ stated that acrylic appliances that were cleaned solely with water had more microorganisms than the ones that were cleaned with antibacterial agents. Therefore immersing the appliance in chemical cleansers such as alkaline peroxide and sodium hypochlorite is highly recommended.

The effects of chemical cleansers on different properties of acrylic bases such as surface roughness, color stability, dimensional changes, etc should also be noticed.

In a study conducted by Felipucci et al. ${ }^{9}$ in 2011 the effects of different cleansers on surface characteristics of removable dentures were evaluated and they showed that Corega ${ }^{\circledR}$ solution neither increases surface roughness of resins nor tarnishes the metal surfaces.

Although the effects of chemical cleansers on surface characteristics of acrylic bases are widely investigated, little information on their effects on dimensional changes of orthodontic appliances is available. Considering the importance of appliance adaptation and its dimensional stability, the purpose of this study was to evaluate the effects of Corega ${ }^{\circledR}$ solution on dimensional changes of orthodontic appliances in comparison with two conditions including dry and water environment.

\section{MATERIALANDMETHODS}

Sixty six acrylic resin specimens were made in quadrangular metal mold with internal space of $57 \mathrm{~mm} \times 20 \mathrm{~mm} \times 3 \mathrm{~mm}$. The mold was filled with self cure acrylic resin (Orthoresin, Dentsply, Victoria, Australia) in single increment. A glass microscopic slide was placed over the mold and pressed to remove excessive resin. All specimens were put in $60^{\circ} \mathrm{C}$ water to increase polymerization and reduce porosity formation. This also resulted in increasing the yield strength and transverse strength of resin.

After the setting time, the specimens were removed carefully from the mold. The length, width and diameter of each specimen were measured right after removal from the mold with digital caliper (Mitutoyo, Kawasaki, Kanagawa, Japan) with measurement accuracy of $0.01 \mathrm{~mm}$. Specimens with no porosity or distortion were chosen and randomly divided into three groups. The samples in the first group were kept in dry environment, the samples in the second group were stored in water and the 
samples in the third group were stored in Corega ${ }^{\circledR}$ solution (sodium bicarbonate, citric acid, lactose, sodium carbonate, sodium lauryl sulfate, PVP/VA copolymer, mint flavors). All specimens were kept in incubator in $25^{\circ} \mathrm{C}$ as to mimic the room temperature.

Different dimensions of the specimens were blindly measured after 18 hours, 48 hours and 168 hours (one week) by two independent evaluators. After each measurement the specimens were returned to their original environment to reduce errors.

Dimensional change for each specimen was calculated using this formula:

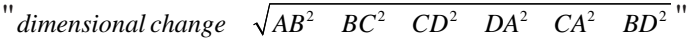

Data were analyzed statistically using

Tukey test and Repeated measures test at a significance level of $5 \%$.

\section{RESULTS}

The median score and dimensional change in all three groups in three different time periods are shown in tables 1,2 and 3 .

Multiple comparison analysis by Tukey Test showed that after 18 hours, dry environment had the highest amount of dimensional change (pvalue: 0.02 ) and was significantly different from water and Corega ${ }^{\circledR}$ solution (p-value :0.008 and 0.046 respectively). No significant difference was seen between dimensional change in water and Corega ${ }^{\circledR}$ solution (p-value: 0.783 ).

After 48 hours, dry environment had the highest amount of dimensional change (p-value: 0.03 ) and was significantly different from water and Corega ${ }^{\circledR}$ solution (p-value: 0.048 and 0.016 respectively). No significant difference was seen between dimensional changes in water and Corega ${ }^{\circledR}$ solution (p-value: 0.901).

After 168 hours (1 week), dry environment had the highest amount of dimensional change (p-value: 0.04) and was significantly different from water and Corega ${ }^{\circledR}$ solution (p-value: 0.009 and 0.004 respectively). No significant difference was seen between dimensional change in water and Corega ${ }^{\circledR}$ solution (p-value: 0.825).

Data analysis by Repeated measures test showed that acrylic resin's dimensional change in
Table 1. Mean dimensional change according to different elapsed time and environment

\begin{tabular}{llll}
\hline & Dry & Water & p-value \\
\hline 18hour & $-0.19(0.216)^{*}$ & $+0.013(0.177)$ & $0.008^{\mathrm{b}}$ \\
48hour & $-0.197(0.29)$ & $-0.001(0.236)$ & $0.048^{\mathrm{b}}$ \\
168hour & $-0.193(0.164)$ & $+0.028(0.179)$ & $0.009^{\mathrm{b}}$ \\
p-value & $0.06^{\mathrm{a}}$ & $0.065^{\mathrm{a}}$ & - \\
\hline
\end{tabular}

Notes: negative values stand for shrinkage.

*(standard deviation)

a : non-significant

b: significant

Table 2. Mean dimensional change according to different elapsed time and environment

\begin{tabular}{llll}
\hline & Dry & Corega ${ }^{\circledR}$ solution & p-value \\
\hline 18hour & $-0.19(0.216)^{*}$ & $-0.031(0.251)$ & $0.046^{\mathrm{b}}$ \\
48hour & $-0.197(0.29)$ & $+0.034(0.274)$ & $0.016^{\mathrm{b}}$ \\
168hour & $-0.193(0.164)$ & $+0.061(0.213)$ & $0.004^{\mathrm{b}}$ \\
p-value & $0.06^{\mathrm{a}}$ & $0.07^{\mathrm{a}}$ & - \\
\hline
\end{tabular}

Notes: negative values stand for shrinkage.

*(standard deviation)

a : non-significant

b: significant

Table 3. Mean dimensional change according to different elapsed time and environment

\begin{tabular}{llll}
\hline & Corega ${ }^{\circledR}$ solution Water & p-value \\
\hline 18hour & $-0.031(0.251)^{*}$ & $+0.013(0.177)$ & $0.783^{\mathrm{a}}$ \\
48hour & $+0.034(0.274)$ & $-0.001(0.236)$ & $0.901^{\mathrm{a}}$ \\
168hour & $+0.061(0.213)$ & $+0.028(0.179)$ & $0.825^{\mathrm{a}}$ \\
p-value & $0.07^{\mathrm{a}}$ & $0.065^{\mathrm{a}}$ & - \\
\hline
\end{tabular}

Notes: negative values stand for shrinkage.

*(standard deviation)

a : non-significant

b: significant

dry environment over different periods of time is not statistically significant (p-value $>0.05$ ). Also no significant difference in dimensional changes was observed in water and Corega ${ }^{\circledR}$ solution over the same periods of time.

\section{DISCUSSION}

Patient's compliance with the use of orthodontic appliances depends on not just internal 
and external motivation but other factors such as appliance type, appliance convenience for the patient, lack of tissue soreness and its biocompatibility. Orthodontic appliances are made from clear orthodontic acrylic resins with the base of polymethyl methacrylate. This material undergoes dimensional changes affected by released stresses, water absorption or dehydration and incomplete polymerization. ${ }^{5}$

Any interruption in regular use of the appliance caused by journey, missing, primary non compliance, or even part time use of the appliance in the retention phase makes the dimensional changes to be more evident in the form of distortion and lack of tissue adaptation when the patient starts to wear the appliance again.

On the other hand, orthodontic appliances increase biofilm accumulation in oral cavity due to their retentive sites (i.e. clasps, springs, etc). Moreover the acrylic base plates can provide ideal niches for microbial growth. Therefore the orthodontic appliances should be kept in a specific environment outside of the mouth to disinfect the appliance and improve oral health.

With respect to these two basic factors i.e. deformities and dimensional changes of the orthodontic acrylic appliances and their crucial influence on oral health and hygiene, the aim of this study was to determine the best environment to keep the orthodontic self cured acrylic appliances in.

Many factors such as size and shape, appliance thickness, stress release, etc affect the dimensional changes of acrylic resins ${ }^{2}$, so it seems practical to use a specimen with a simple shape to study the dimensional changes of acrylic resins. This way we can eliminate other factors and attribute the results of dimensional changes solely to the acrylic resins and the methods used.

In this study we used rectangular shaped specimens with controlled thickness. Therefore we used a norm to investigate these dimensional changes. Four vertices of the rectangle were named as $A, B, C$ and $D$ and the resultant six vectors were analyzed as $N_{0 r m}=\sqrt{A B^{2}+B C^{2}+C D^{2}+D A^{2}+C A^{2}+B D^{2}}$ that would be a simple method to compare the dimensional changes of rectangular specimens.

As stated before the dry environment caused a significant polymerization shrinkage (0.190 ) after 18 hours that seemed to be due to the primary polymerization shrinkage and the continued setting shrinkage of the acrylic resins. This outcome obtained in dry environment is accordant with previous studies that mentioned that the highest dimensional changes (shrinkage) occur in the first 24 hours. Also Mojon et al. ${ }^{10}$ showed that $80 \%$ of the dimensional changes in the room temperature occur in the first 17 minutes.

This shrinkage continued for 48 hours at a lower rate $(-0.197)$ that can be due to the polymerization cycles.

After one week only a small portion of the shrinkage was compensated $(-0.193)$ that seemed to be due to humidity absorption. Also Polychronakis et al. ${ }^{3}$ showed that weight changes of the resins kept in dry environment with the humidity of $100 \%$ after a certain period of time equal the changes of those kept in water environment that this can explain the reason of shrinkage compensation occurred in our study.

In water environment the amount of water absorption is more than the amount of shrinkage and changes are in the form of expansion $(+0.013)$ which is not significant. Past studies show shrinkage in 24 hours $^{11}$, this disagreement can be due to difference in residual monomers in cold cure and heat cure acrylic resins and also different diffusion coefficients.

After 48 hours the amount of shrinkage exceeds the amount of expansion and the overall change becomes a non significant shrinkage (0.001). After one week, progressive water absorption caused a non significant net expansion (+0.028). The studies conducted by Chen et al. ${ }^{12}$ and Goodkind et al. ${ }^{13}$ also had the same results and showed no significant changes after one week of being kept in water environment. However Miessi et al. ${ }^{14}$ showed that when specimens are kept in water for longer periods of time (2 months) significant expansion may occur and it would damage appliance fitness. This further expansion is caused by the great capacity of resin for water sorption.

In Corega ${ }^{\circledR}$ solution after 18 hours, changes occur in the form of non significant shrinkage (-0.031) that could be due to the amount of shrinkage in the first hours after polymerization. The difference between Corega ${ }^{\circledR}$ solution and water in the first 18 hours may be due to the different amount of monomer release because of 
different components of Corega ${ }^{\circledR}$ solution and water.

After 48 hours water absorption exceeds the amount of shrinkage (0.034) and continues to increase after a week (0.061) but none of these amounts of expansion are significant.

The mean dimensional changes after 18 hours in dry environment, water and Corega ${ }^{\circledR}$ solution were $-0.190,+0.013$ and -0.031 respectively that suggests water environment has caused minimum changes and dry environment has caused maximum changes. Ghanbarzadeh et al. ${ }^{15}$ reported equal amount of changes in dry environment and water after 24 hours that this non concurrence could be due to use of different types of acrylic resins.

The mean dimensional changes after 48 hours in dry environment, water and Corega ${ }^{\circledR}$ solution were $-0.197,-0.001$ and +0.034 respectively that showed water environment has caused minimum changes and dry environment has caused maximum changes. The study conducted by Ghanbarzadeh et al. ${ }^{15}$ also confirmed the same results.

The mean dimensional changes after 1 week in dry environment, water and Corega ${ }^{\circledR}$ solution were $-0.192,+0.028$ and +0.061 respectively that shows again water environment has caused minimum changes and dry environment has caused maximum changes.

Ghanbarzadeh et al. ${ }^{15}$ investigated the effects of storage conditions on dimensional changes of acrylic post-core patterns and concluded that among 3 different conditions (dry atmosphere at $25^{\circ} \mathrm{C}, 100 \%$ humidity at $25^{\circ} \mathrm{C}$ and water at $4^{\circ} \mathrm{C}$ ) $100 \%$ humidity at $25^{\circ} \mathrm{C}$ is the best environment that causes minimal changes in dimensions.

They also found that storage time affected the post thickness but did not have any effects on the length or thickness of the core. Also in our study the storage time was not effective (pvalue $>0.05$ ). However Miessi et al. ${ }^{14}$ reported that the storage time had effects on dimensional changes. The result of this study suggests that keeping the appliance in dry environment should be avoided due to the highest amount of dimensional changes. Dimensional changes are not significantly different between water and Corega ${ }^{\circledR}$ solution but Corega ${ }^{\circledR}$ solution is the superior choice due to its antibacterial effects.

\section{CONCLUSION}

Orthodontic appliances should be kept in water or Corega ${ }^{\circledR}$ solution and Corega ${ }^{\circledR}$ solution is the superior choice due to its antibacterial effects.

\section{Conflicts of interest}

The authors deny any conflicts of interest related to this study.

\section{REFERENCES}

1. Wong, D.M., Cheng, L.Y., Chow, T.W., Clark, R.K. Effect of processing method on the dimensional accuracy and water sorption of acrylic resin dentures. J Prosthet Dent. 1999; 81: 300-4.

2. Salim, S., Sadamori, S., Hamada, T. The dimensional accuracy of rectangular acrylic resin specimens cured by three denture base processing methods. J Prosthet Dent. 1992; 67: 879-81.

3. Polychronakis, N., Yannikakis, S., Zissis, A. A clinical 5-year longitudinal study on the dimensional changes of complete maxillary dentures. Int J Prosthodont. 2003; 16: 78-81.

4. Dixon, D.L., Breeding, L.C., Ekstrand, K.G. Linear dimensional variability of three denture base resins after processing and in water storage. J Prosthet Dent. 1992; 68: 196-200.

5. Consani, R.L., Azevedo, D.D., Mesquita, M.F., Mendes, W.B., Saquy, P.C. Effect of repeated disinfections by microwave energy on the physical and mechanical properties of denture base acrylic resins. Braz Dent J. 2009; 20:1327.

6. Lessa, F.C., Enoki, C., Ito, I.Y., Faria, G., Matsumoto, M.A., Nelson-Filho, P. In-vivo evaluation of the bacterial contamination and disinfection of acrylic baseplates of removable orthodontic appliances. Am J Orthod Dentofacial Orthop. 2007; 131: 705.e11-7.

7. Zabokova-Bilbilova, E., Popovska, L., Kapusevska, B., Stefanovska, E. White spot lesions: prevention and management during the orthodontic treatment. Prilozi. 2014; 35: 161-8.

8. Zarb, G.A., Hobkirk, J., Eckert, S., Jacob, R. Prosthodontic treatment for edentulous patients: complete dentures and implantsupported prostheses: Elsevier Health Sciences; 2013.

9. Felipucci, D.N., Davi, L.R., Paranhos, H.F., Bezzon, O.L., Silva, R.F., Pagnano, V.O. Effect of different cleansers on the surface of removable 
partial denture. Braz Dent J. 2011; 22: 392-7.

10. Mojon, P., Oberholzer, J.P., Meyer, J.M., Belser, U.C. Polymerization shrinkage of index and pattern acrylic resins. J Prosthet Dent. 1990; 64: 684-8.

11. Shukor, S.S., Juszczyk, A.S., Clark, R.K., Radford, D.R. The effect of cyclic drying on dimensional changes of acrylic resin maxillary complete dentures. J Oral Rehabil. 2006; 33: 654-9.

12. Chen, J.C., Lacefield, W.R., Castleberry, D.J. Effect of denture thickness and curing cycle on the dimensional stability of acrylic resin denture bases. Dent Mater. 1988; 4: 20-4.
13. Goodkind, R.J., Schulte, R.C. Dimensional accuracy of pour acrylic resin and conventional processing of cold-curing acrylic resin bases. $J$ Prosthet Dent. 1970; 24(6): 662-8.

14. Miéssi, A.C., Goiato, M.C., dos Santos, D.M., Dekon, S.F., Okida, R.C. Influence of storage period and effect of different brands of acrylic resin on the dimensional accuracy of the maxillary denture base. Braz Dent J. 2008; 19(3): 204-8.

15. Ghanbarzadeh, J., Sabooni, M., Roshan-Nejad, $\mathrm{R}$. The effect of storage conditions on dimensional changes of acrylic post-core patterns. J Dent (Tehran). 2007; 4: 27-31. 